\title{
The modified hole board as a differential screen for behavior in rodents
}

\author{
F. OHL, F. HOLSBOER, and R. LANDGRAF \\ Max Planck Institute of Psychiatry, Munich, Germany
}

\begin{abstract}
We describe a modified hole board ( $\mathrm{mHB}$ ) paradigm as a test for unconditioned behavior in rodents that is aimed at analyzing a variety of behavioral dimensions. We demonstrate that the mHB enables the investigation of different behavioral dimensions in rodents in only one test by reproducing the behavioral characteristics previously collected from multiple behavioral tests in rats bred for either high or low anxiety-related behavior. In addition, the test design, which allows the experimental animal to maintain social contact with its group mates during the test, was shown to minimize stress for experimental animals and, moreover, to enable the investigator to assess social affinity among group mates. In summary, the mHB test enables animal models to be comprehensively phenotyped, while simultaneously reducing the number of animals and the amount of time required. Therefore, the mHB represents an alternative to the common practice of using a series of more specific tests.
\end{abstract}

During recent years, biomedical research has made rapid progress, and neurobiological and genetic sciences today are able to produce a high number of potential animal models and novel compounds. To investigate the underlying neurobiological mechanisms of disorders to be modeled, to characterize those animal models, and to evaluate behavioral effects of potential pharmacological treatment strategies, behavioral tests are essential. In general, test procedures are predictive of one specific type of behavioral dimension, and, therefore, several independent tests have to be performed to dissociate behavioral alterations or effects (Escorihuela et al., 1999; Henniger et al., 2000). In order to avoid interference between distinct tests (Belzung \& LePape, 1994), each of the tests should be performed with naive experimental animals. Consequently, such a multiple test battery results not only in a time- and money-consuming procedure but also in a high number of animals needed.

Studies based on behavioral tests that are focused on a more detailed ethological analysis of experimental animals in a single complex paradigm may overcome these disadvantages (Cruz, Frei, \& Graeff, 1994; Lister, 1990; Rodgers, Cao, Dalvi, \& Holmes, 1997; Wilson, 2000). Therefore, we aimed at developing a test paradigm that would allow a variety of behavioral dimensions to be investigated in one test. In addition, the test was designed to minimize stress by allowing the experimental animal to maintain social contact with its group mates during the

We thank Alexandra Wigger for the management of the HAB/LAB breeding program and Albin Varga for animal care. The skilled technical assistance of Monika Rücker is gratefully acknowledged. This work was partly supported by the Volkswagen-Stiftung to F.O. (I/76268). Correspondence concerning this article should be addressed to F. Ohl, Max Planck Institute of Psychiatry, Kraepelinstr. 2, D-80804 Munich, Germany (e-mail: ohl@mpipsykl.mpg.de). test, thus circumventing stressful social isolation (Ahmed, Stinus, Lemoal, \& Cador, 1995; Kim \& Kirkpatrick, 1996).

The modified hole board ( $\mathrm{mHB}$ ) paradigm represents a test for unconditioned behavior and essentially comprises the characteristics of a hole board and an open field test. Although the original hole board was designed to investigate exploration in rodents (File \& Wardill, 1975; for review, see Lister, 1990), the mHB also allows for a variety of other behavioral dimensions to be investigated. To establish the mHB test, we investigated the behavior of Wistar rats that had been bred selectively for innate emotionality on the elevated plus maze (EPM; Liebsch, Linthorst, et al., 1998). In previous studies, it was demonstrated that the line of high anxiety-related behavior rats (HABs) show an increased innate emotionality and more passive stress coping strategies than the line of low anxiety-related behavior rats (LABs) under stressful conditions (Liebsch, Linthorst, et al., 1998; Liebsch, Montkowski, Holsboer, \& Landgraf, 1998). Extensive studies, including pharmacological validation, have demonstrated that hyperanxiety in HABs represents a robust trait, resembling the signs and symptoms seen in psychiatric patients (Keck et al., 1999; Landgraf, Wigger, Holsboer, \& Neumann, 1999; Liebsch,Linthorst, et al., 1998). Thus, HABs represent a unique animal model of innate anxiety with face, predictive, and construct validity.

By investigating these rat lines in the $\mathrm{mHB}$, we aimed at demonstrating that the behavioral characteristics found in multiple behavioral tests could be reproduced by using only one test. Furthermore, we compared the behavior of animals tested singly and in groups in the $\mathrm{mHB}$ in order to evaluate whether or not the design of the $\mathrm{mHB}$ minimized stress for experimental animals. In addition, this comparison should show whether group-wise testing in the $\mathrm{mHB}$ allows for the assessment of social affinity among group mates. 


\section{METHOD}

\section{Animals}

A detailed description of the selection and the breeding of both HABs and LABs was given previously in detail by Liebsch, Montkowski, et al. (1998). Both rat lines, derived from Wistar rats, were kept and bred in the animal facilities of the Max Planck Institute under standard laboratory conditions (12:12 light:dark, lights on at $6: 00,22^{\circ} \mathrm{C}, 60 \%$ humidity and free access to water and standard rat chow), and, over the years, HABs and LABs have been treated identically in terms of care, mating, and behavioral testing. The animals used in the present study were adult male individuals (weight: 280-350 g) from the F10 generation. As an additional control group, Wistar rats were purchased (normal anxiety-related behavior rats, NABs; Charles River Laboratories, Sulzfeld, Germany; $n=12$ ) from the same stock as the HABs and LABs. To investigate the effects of chronic social isolation on the behavior displayed in the mHB, male HABs $(n=6)$ were housed singly (cage size: $38 \times 25$ $\times 20 \mathrm{~cm})$ for 6 weeks. All other rats $(n=34)$ were housed in groups of $3-5$ in standard rat cages $(58 \times 38 \times 20 \mathrm{~cm})$.

\section{Modified Hole Board (mHB)}

The mHB comprises the characteristics of a hole board, originally designed to investigate exploration in rodents (File \& Wardill, 1975; for a review, see Lister, 1990), and an open field, a test paradigm that is highly standardized to evaluate locomotor activity (for a review, see Kelley, 1993). The hole board setup is based on a previously modified hole board that was designed to evaluate cognitive functions in tree shrews (Ohl \& Fuchs, 1999; Ohl, Oitzl, \& Fuchs, 1998 ) and that was adapted for investigations in rodents. Figures 1 and 2 include schematic drawings of the modif ied hole board.
The board consisted of opaque gray PVC $(60 \times 20 \times 2 \mathrm{~cm})$ with 23 holes $(1.5 \times 0.5 \mathrm{~cm})$ staggered in three lines. All holes on the board were covered by movable lids of the same material. The board was placed in the middle of a PVC box $(100 \times 50 \times 50 \mathrm{~cm})$, thus representing the central area of an open field. The outer area was divided into 12 quadrats $(20 \times 16 \mathrm{~cm})$ by white lines. The PVC box was enlarged by an additional compartment $(50 \times 50 \times 50 \mathrm{~cm})$, in which the group mates of the experimental animal were placed during the test period (Figures 1 and 2). The group compartment was separated from the experimental compartment by a transparent PVC partition perforated with 120 holes $(1 \mathrm{~cm}$ in diameter).

\section{Analysis and Measures}

All tests were performed with naive male rats. To investigate line differences, HABs, NABs, and LABs (HABs and NABs: $n=12$ each; LABs: $n=13$ ) were housed and tested by group. Behavioral data derived from $\mathrm{HABs}$ in the first part of the study were also included in the second part (i.e., the comparison of housing and experimental conditions) as group. Two additional groups of HABs were housed by group but were tested singly in the mHB (single; $n=9$ ). For the investigation of the effects of chronic social isolation on the behavior displayed in the mHB, 6 male HABs (isolated) were housed singly for 6 weeks and tested singly.

All experiments were carried out during the light period between 12:00 and 16:00. One week prior to behavioral testing, the animals were transferred to the testing room for habituation. For group-wise testing in the $\mathrm{mHB}$, all animals belonging to one social group (i.e., group mates) were placed in the group compartment. After 30 min of habituation, each of the animals was successively placed into the experimental box (for start position, see Figure 1). For individual testing, each animal was transferred directly from the home cage to the experimental compartment.

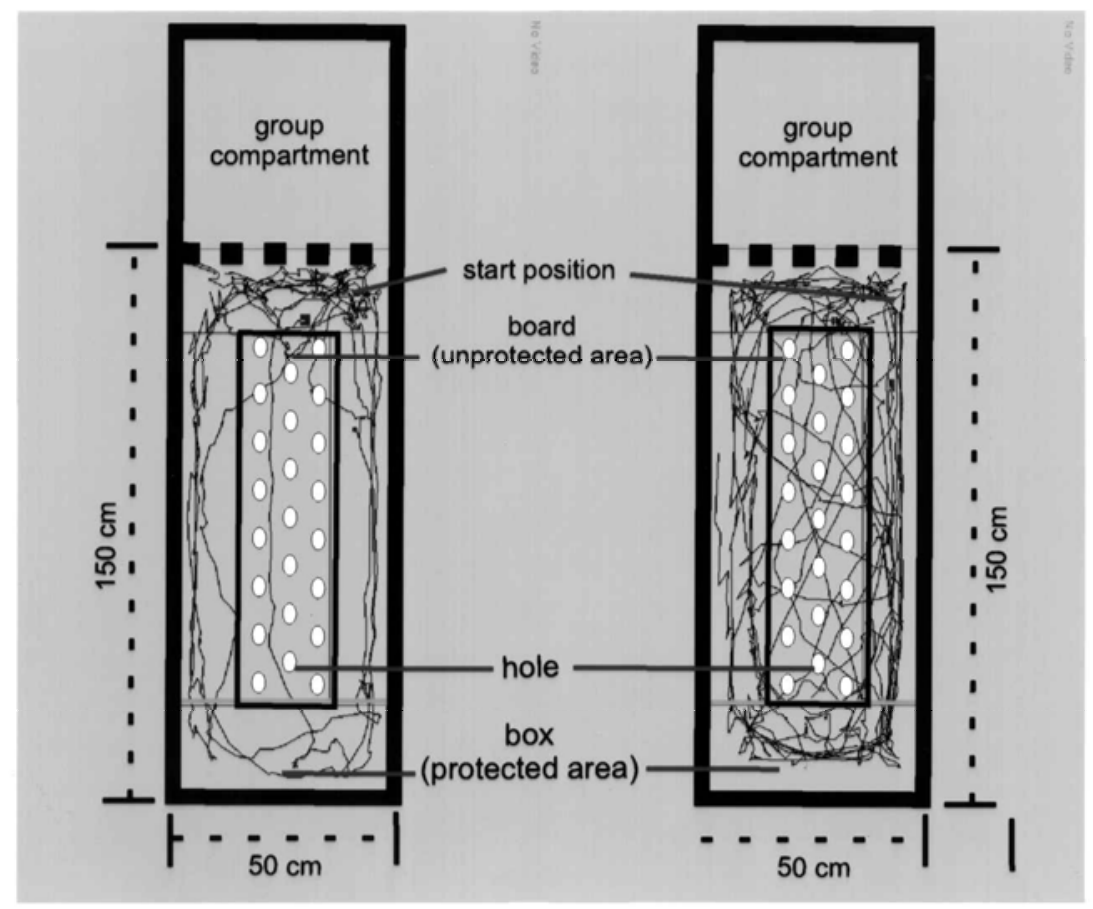

HAB rats

LAB rats

Figure 1. The activity pattern of high anxiety-related behavior rats and low anxietyrelated behavior rats in the modified hole board clearly shows the differences in avoidance behavior towards the unprotected area (i.e., anxiety) between the two lines. 

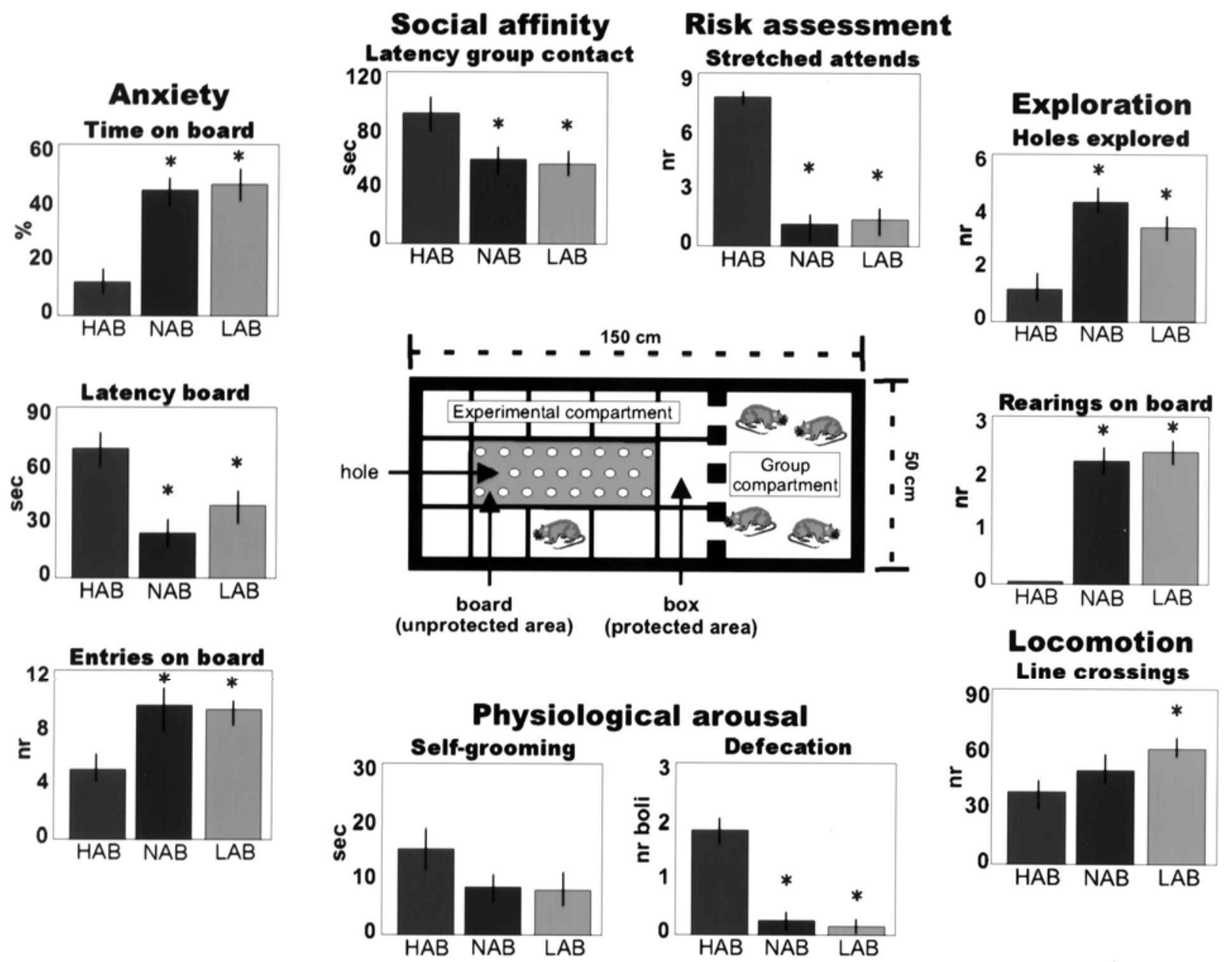

Figure 2. Data (mean $\pm S E M$; HAB, $n=12 ; \mathrm{NAB}, n=12 ; \mathrm{LAB}, n=13)$ from behavioral parameters (one-way ANOVA) measured using the modified hole board test $(* p<.05 \mathrm{vs}$. HABs) and a schematic drawing of the test setup (figure partially modified from Ohl et al., 2001).

Each animal was placed at the start position in one corner close to the group compartment facing the board (see Figure 1) and was tested for $5 \mathrm{~min}$ in moderate light conditions (30 Lux). The experimental box was cleaned with warm water after every test. All tests were videotaped and tracked by Ethovision (Noldus, Wageningen, $\mathrm{NL}$ ) and were directly monitored from behind the group compartment by the use of the Observer (Noldus, Wageningen, NL) by a trained observer blind to the rat line.

Parameters were measured and assigned to different behavioral categories following the results of previous studies (Ohl, Sillaber, Binder, Keck, \& Holsboer, 2001; Ohl, Toschi, Wigger, Henniger, \& Landgraf, 2001) and are shown in Table 1.

\section{Statistics}

The behavioral data from the basal test in HABs, NABs, and LABs were analyzed by an one-way analysis of variance (ANOVA; STATISTICA, StatSoft, Tulsa, USA) with rat line as the between-groups factor followed by a post hoc Newman-Keuls test where appropriate. Due to the lower number of animals per group, the comparison of group and singly tested animals was performed by Kruskal-Wallis ANOVA by ranks, followed by nonparametric Mann-Whitney-U test (STATISTICA, StatSoft, Tulsa, USA) $(p<.05$ was accepted as significant). Data are presented as means $( \pm S E M)$.

\section{RESULTS}

The comparison of the basal behavior of HABs, NABs, and LABs (Figure 2) revealed significant line differences in terms of avoidance behavior toward the unprotected area [i.e., anxiety; time on board, $F(2,35)=30.1, p<$ .001 ; latency board, $F(2,35)=10.8, p<.001$; entries on board, $F(2,35)=22.8, p<.001]$ and also for risk assessment behavior [stretched attends, $F(2,35)=60.3$, $p<.001]$. In addition, locomotor activity [line crossings, $F(2,35)=4.1, p<.05]$ and exploration of the unprotected area [rearings on board, $F(2,35)=8.1, p<.01$; holes explored, $F(2,35)=9.4, p<.001]$ showed significant effects for the factor line. Finally, rat lines differed in terms of social affinity [group contact, $F(2,35)=9.7, p<.001$ ] and defecation (boli, $F(2,35)=8.35, p<.005]$. No difference was found for exploration of the protected area [rearings box, $F(2,35)=1.2$, n.s., data not shown) or for grooming $[F(2,35)=3.0 ;$ n.s. $]$. The results of the posthoc analysis are presented in Figure 2. 
Table 1

Parameters Measured and Assigned to the Different Behavioral Categories

Avoidance Behavior Toward an Unprotected Area (i.e., Board), Representing Anxiety

Percent time on the board

Latency to the first board entry (i.e., entering the board with at least both forepaws)

Number of board entries

Exploration-Related Behavior

Holes explored (i.e., sniffing at a hole or actively moving a lid)

Rearings on the board

Rearings in the box

Risk Assessment Behavior

Stretched body posture

Locomotor Activity

Line crossings in the box (i.e., crossing a line with all four paws)

Social Affinity of the Experimental Animal Toward Its Groups Mates Latency to the first group contact (i.e., rearing or sniffing at the partition)

To control if the animals were attracted by the partition per se, the percentage of time spent at the partition was also calculated for the second part of the experiment (group vs. single vs. isolated)

Physiological Arousal

Latency to the first self-grooming

Defecation

The results of the comparison of group-housed/groupwise tested, group-housed/singly tested and isolated/ singly tested HABs are listed in Table 2. Singly tested HABs differed from group tested HABs, as they revealed significantly increased anxiety-related behavior, arousal, and risk assessment behavior in the $\mathrm{mHB}$. Chronically isolated HABs showed comparable differences, as compared with group tested animals, and, in addition, they revealed significantly increased exploratory behavior. The comparison of singly tested versus isolated animals showed that the latter were more aroused but also displayed an increased hole exploration, as compared with the former.

\section{DISCUSSION}

The mHB essentially comprises the characteristics of a hole board and an open field. By investigating the basal behavior of rats bred for either high or low anxietyrelated behavior and, in addition, normal Wistar rats, we demonstrated that the $\mathrm{mHB}$ allows for the detection of alterations in a wide range of behaviors, including anxietyrelated behavior, risk assessment, exploration, locomotor activity, arousal, and social affinity.

The results of the comparison between rat lines in the $\mathrm{mHB}$ test (Figure 2) matches the finding s of previous results obtained by different tests of unconditioned behavior, such as the EPM (Liebsch, Montkowski, et al., 1998), the open field (Henniger et al., 2000), and the dark-light box (Henniger et al., 2000), which showed that HABs and LABs differ in terms of anxiety-related behavior. Also, a reduction in exploration of unprotected areas (i.e., board) in HABs was found, whereas no differences in exploration of the protected area (i.e., rearings in the box) emerged among the three groups. Since differences between rat lines in exploration-related behavior were limited to the unprotected area and no significant differences were found between LABs and NABs, it might be suggested that exploration-related alterations in $\mathrm{HABs}$ are anxiety driven. These findings show that behavioral characteristics previously collected from multiple tests could be reproduced by using only one comprehensive paradigm.

As in the $\mathrm{mHB}$, since both an increase in anxietyrelated behavior and a reduction in locomotor activity occurred in HABs (see Figure 1), it is, at the first sight,

Table 2

Results of the Comparison Between the Behavior Displayed in the $\mathrm{mHB}$ by Group-tested, Singly Tested, and Chronically Isolated HABs

\begin{tabular}{|c|c|c|c|c|c|c|c|c|}
\hline \multirow[b]{2}{*}{ Behavior } & \multicolumn{2}{|c|}{ Group } & & \multicolumn{2}{|c|}{ Single } & & \multicolumn{2}{|c|}{ Isolated } \\
\hline & Mean & SEM & & Mean & SEM & & Mean & $S E M$ \\
\hline \multicolumn{9}{|l|}{ Anxiety } \\
\hline Time on board (\%) & 13.8 & 2.6 & $* \dagger$ & 3.3 & 1.1 & & 4.4 & 1.6 \\
\hline Entries on board (No.) & 4.8 & 0.6 & $*$ & 2.6 & 0.8 & & 3.0 & 0.9 \\
\hline Latency first board visit (sec) & 61.7 & 7.6 & & 108.6 & 41.5 & & 66.5 & 45.1 \\
\hline \multicolumn{9}{|l|}{ Exploration } \\
\hline Holes explored (No.) & 1.2 & 0.4 & & 2.0 & 0.7 & & 3.8 & 2.0 \\
\hline Latency hole first visit (sec) & 180.4 & 31.2 & $\dagger$ & 150 & 42.6 & $\ddagger$ & 16.9 & 5.6 \\
\hline Rear box (No.) & 12.2 & 1.7 & & 13.2 & 1.9 & & 15.5 & 3.4 \\
\hline Rear board (No.) & 0.0 & 0.0 & & 0.2 & 0,1 & & 0.0 & 0.0 \\
\hline \multicolumn{9}{|l|}{ Risk assessment } \\
\hline Stretched attends (No.) & 7.9 & 0.6 & $* \dagger$ & 0.0 & 0.0 & $\ddagger$ & 1.5 & 0.8 \\
\hline \multicolumn{9}{|l|}{ Locomotor activity } \\
\hline Line crossings (No.) & 41.1 & 2.1 & & 47.1 & 5.1 & & 53.5 & 9.7 \\
\hline \multicolumn{9}{|l|}{ Social affinity } \\
\hline Time partition (\%) & 34.7 & 4.0 & $* \dagger$ & 19.4 & 4.4 & & 10.8 & 1.9 \\
\hline \multicolumn{9}{|l|}{ Physiological arousal } \\
\hline Latency groom $(\mathrm{sec})$ & 222.9 & 20.7 & $* \dagger$ & 153.1 & 20.8 & $\ddagger$ & 42.3 & 31.3 \\
\hline Boli (No.) & 1.9 & 0.4 & $\dagger$ & 2.4 & 0.6 & $\ddagger$ & 0.3 & 0.2 \\
\hline
\end{tabular}


difficult to decide whether the observed changes in anxiety-related behavior were secondary to a decrease in general activity. However, in a previous study, we showed by factor analysis that anxiety-related behavior and locomotor activity displayed in the $\mathrm{mHB}$ are not causally related (Ohl et al., 2001). These findings indicate that alterations in anxiety-related behavior in HABs, although being paralleled by a behavioral inhibition (i.e., decreased activity), are not caused by changes in locomotor activity.

As noted above, the mHB allows the animals to maintain visual and olfactory contact with each other. To investigate whether this design reduces the stressful factor of social isolation during the test and, moreover, allows for the assessment of the need for social contact, we compared the behavior of group-housed/group tested, group-housed/singly tested, and chronically isolated/ singly tested naive $\mathrm{HABs}$ in the mHB. It should be noted that the group-tested and singly tested conditions did not only differ with respect to group presence but also with respect to the habituation period in the group compartment. The results (see Table 2) show that singly tested HABs displayed increased anxiety-related behavior and were more aroused than rats tested in the presence of their social group, suggesting that contact to group mates in fact minimizes stress during the test.

Moreover, group-tested HABs spent significantly more time at the partition, as compared with singly tested animals. These results show that the animals were not attracted by the partition per se but that the design of the $\mathrm{mHB}$ in fact allowed for the assessment of social affinity. The shorter latency to first group contact seen in HABs can thus be interpreted as increased social affinity in these animals, as compared with NABs and LABs (Figure 2). This finding suggests that social contact during a novel situation is more important for anxious rats than for nonanxious rats, and it can be hypothesized that social contact might be helpful for HABs in coping with the challenge of a novel environment.

\section{Methodological Aspects}

As noted above, most of the behavioral dimensions assessed in the mHB test could also be obtained by using a multiple test battery of other well-established selective tests such as the open field for locomotor activity (Kelley, 1993), the EPM for anxiety-related behavior (Griebel, Rodgers, Perrault, \& Sanger, 1997; Rogers et al., 1999), and the classical hole board for exploration (File \& Wardill, 1975; Sheldon, 1968). However, if the same animals are exposed to such a multiple test battery, the increase in test experience may well influence the behavior of experimental animals (Koide, Moriwaki, Ikeda, Niki, \& Shiroishi, 2000; Montkowski, Poettig, Mederer, \& Holsboer, 1997). Since this experience may differ between different lines, strains, and genetically modified animals, it may induce severe problems, especially for the basal characterization of potential animal models.

Using a multiple test battery, experience-based interference can only be avoided by performing each test with naive animals, a method that drastically increases the number of animals needed. Furthermore, the behavior of rodents is context dependent, and, when characterizing novel animal models, distinct behavioral tests frequently produce conflicting results in that one behavioral dimension displayed by the same strain differs between distinct tests (Rodgers, 1997; Trullas \& Skolnick, 1993). Such results point toward the need for simultaneous investigations of a high number of behavioral dimensions in one test procedure, an option that is offered by the mHB test.

Recently, we showed that the mHB can also be used for behavioral phenotyping in mice and for pharmacological screening (Ohl, Sillaber, et al., 2001). We tested two mouse strains, C57BL/6 and BALB, for which differences in terms of anxiety are consistently reported, but with varying rank orders between different tests and studies (Avgustinovich, Lipina, Bondar, Alekseyenko, \& Kudryavtseva, 2000; Rogers et al., 1999). In the mBH, no significant differences were found between the two strains in terms of avoidance of the unprotected area. However, the pathways performed by the two strains in the mHB showed that the spatiotemporal parameters are determined by differences in the exploratory strategy of these strains. From these exploratory strategies, it could be suggested that the nonemotional characteristic displayed by BALB mice in more specific tests does not indicate decreased anxiety but may depend on their home base strategy and less pronounced thigmotaxis in comparison with C57BL/6 mice. According to these findings, the results of selective behavioral testing may easily be misinterpreted, whereas the comprehensive analysis of all behavioral dimensions displayed by an animal allows behavioral characteristics and, thus, also pharmacological effects on behavior, to be reliably identified.

In summary, by using the mHB test, it is possible to comprehensively analyze the behavior in rodents and, thus, to characterize potential animal models that mimick a psychopathological condition. At the same time, the $\mathrm{mHB}$ allows the number of animals and the time needed to perform behavioral phenotyping to be reduced. Since the mHB allows a wide range of behavioral parameters to be investigated, alterations in anxiety, exploration, arousal, social affinity, and locomotor activity can be identified, and so, this test procedure represents an alternative to the common practice, of performing a series of more specific tests.

\section{REFERENCES}

Ahmed, S. H., Stinus, L., Le Moal, M., \& Cador, M. (1995). Social deprivation enhances the vulnerability of male Wistar rats to stressorand amphetamine-induced behavioral sensitization. Psychopharmacology, 117, 116-124.

Avgustinovich, D. F., Lipina, T. V., Bondar, N. P., Alekseyenko, O. V., \& Kudryavtseva, N. N. (2000). Features of the genetically defined anxiety in mice. Behavioral Genetics, 30, 101-109.

Belzung, C., \& Le PAPE, G. (1994). Comparison of different behavioral test situations used in psychopharmacology for measurements of anxiety. Physiology \& Behavior, 56, 623-628.

Cruz, A. P. M., Frei, F., \& Graeff, F. G. (1994). Ethopharmacological 
analysis of rat behavior on the elevated plus-maze. Pharmacology, Biochemistry \& Behavior, 49, 171-176.

Escorihuela, R. M., Fernandez-Teruel, A., Gil, L., Aguilar, R. Tobena, A., \& Driscoll, P. (1999). Inbred Roman high- and lowavoidance rats: Differences in anxiety, novelty-seeking, and shuttlebox behavior. Physiology \& Behavior, 67, 19-26.

FiLe, S. E., \& WARDILL, A. G. (1975). Validity of head-dipping as a measure of exploration in a modified hole-board. Psychopharmacologia, 44, 53-59.

Griebel, G., Rodgers, R. J., Perrault, G., \& Sanger, D. J. (1997). Risk assessment behavior: Evaluation of utility in the study of 5-HTrelated drugs in the rat elevated plus-maze test. Pharmacology, Biochemistry \& Behavior, 57, 817-827.

Henniger, M. S. H., Ohl, F., Hölter, S. M., Weissenbacher, P., Toschi, N., Lörscher, P., Wigger, A., SPANAgEL, R, \& LANDGRAF, R. (2000). Unconditioned anxiety and social behaviour in two rat lines selectively bred for high and low anxiety-related behaviour. Behavioural Brain Research, 111, 153-163.

Keck, M. E., Wigger, A., Gesing, A., Welt, T., Reul, J. M. H. M., Holsboer, F., Landgraf, R., \& Neumann, I. D. (1999). The combined DEX/CRH test in high anxiety rats: Involvement of endogenous vasopressin. Society for Neuroscience Abstracts, 64, 13.

Kelley, A. E. (1993). Locomotor activity and exploration. In A. Sahgal (Ed.), Behavioural neuroscience: A practical approach (pp. 121). Oxford: Oxford University Press.

KIM, J. W., \& KirK PATricK, B. (1996). Social isolation in animal models of relevance to neuropsychiatric disorders. Biological Psychiatry, 40, 918-922.

Koide, T., Moriwaki, K., Ikeda, K., NiKi, H., \& Shiroishi, T. (2000). Multi-phenotype behavioral characterization of inbred strains derived from wild stocks of Mus musculus. Mammalian Genome, 11, 664-670.

Landgraf, R, Wigger, A. Holsboer, F., \& Neumann, I. D. (1999). Hyper-reactive hypothalamus-pituitary-adrenocorticalaxis in rats bred for high anxiety-related behaviour. Journal of Neuroendocrinology, 11, 405-407.

Liebsch, G., Linthorst, A. C. E., Neumann I. D., Reul, J. M. H. M., Holsboer, F., \& LANDGRAF, R. (1998). Behavioral, physiological, and neuroendocrine stress response and differential sensitivity to diazepam in two Wistar rat lines selectively bred for high- and lowanxiety-related behavior. Neuropsychopharmacology, 19, 381-396.

Liebsch, G., Montkowski, A., Holsboer, F., \& Landgraf, R. (1998).
Behavioural profiles of two Wistar rat lines selectively bred for high or low anxiety-related behaviour. Behavioural Brain Research, 94 301-310.

LISTER, R. G. (1990). Ethologically based animal models of anxiety disorders. Pharmacological Therapy, 46, 321-340.

Montrowski, A., Poettig, M., Mederer, A., \& Holsboer, F. (1997). Behavioural performance in three substrains of mouse strain 129. Brain Research, 762, 12-18.

OHL, F., \& Fuchs, E. (1999). Differential effects of chronic stress on memory processes in the tree shrew. Cognitive Brain Research, 7, 379-387.

Oht, F., Ortzl, M. S., \& Fuchs, E. (1998). Assessing cognitive functions in tree shrews: Visuo-spatial and spatial learning in the home cage. Journal of Neuroscience Methods, 81, 35-40.

Ohl, F., Sillaber, I., Binder, E., Keck, M. E.. \& Holsboer, F. (2001). Differential analysis of basal behavior and diazepam-induced alterations in $\mathrm{C} 57 \mathrm{BL} / 6$ and $\mathrm{BALB} / \mathrm{c}$ mice using the modified hole board. Journal of Psychiatric Research, 35, 147-154.

Ohl, F., Toschi, N., Wigger, A., Henniger, M. S. H., \& LANDgraf, R. (2001). Dimensions of emotionality in a rat model of innate anxiety. Behavioral Neuroscience, 115, 429-436.

RodGers, R. J. (1997). Animal models of "anxiety": Where next? Behavioural Pharmacology, 8, 477-496.

Rodgers, R. J, CaO, B.-J., Dalvi, A., \& Holmes, A. (1997). Animal models of anxiety: An ethological perspective. Brazilian Journal of Medical \& Biological Research, 30, 289-304.

Rogers, D. C., Jones, D. N. C., Nelson, P. R., Jones, C. M., Quilter, C. A., Robinson, T. L., \& Hagan J. J. (1999). Use of SHIRPA and discriminant anaylsis to characterise marked differences in the behavioural phenotype of six inbred mouse strains. Behavioural Brain Research, 105, 207-217.

SHELDON, M. H. (1968). Exploratory behaviour: The inadequacy of activity measures. Psychological Science, 11, 38 .

Trullas, R., \& Skolnick, P. (1993). Differences in fear motivated behaviors among inbred mouse strains. Psychopharmacology, 111, 323-331.

WiLson, J. H. (2000). A conspecific attenuates prolactin responses to open-field exposure in rats. Hormones \& Behavior, 38, 39-43.

(Manuscript received October 4, 2000; revision accepted for publication May 25, 2001.) 